\title{
Relación entre la adquisición del gateo y la marcha independiente en el niño de alto riesgo en seguimiento
}

\author{
Ma. del Pilar Ibarra-Reyes ${ }^{1 *}$, Martina A. Guido-Campuzano², Luis A. Fernández-Carrocera³, \\ Rosalía Jiménez-Quiroz ${ }^{4}$, Ma. de la Paz Conde-Reyes ${ }^{5}$, Patricia García-Alonso-Themann², \\ Carlos Martínez-Cruz ${ }^{6}$ y René H. Barrera-Reyes ${ }^{7}$
}

${ }^{1}$ Estimulación Neuromotora, Departamento de Seguimiento Pediátrico; ${ }^{2}$ Pediatría, Departamento de Seguimiento Pediátrico; ${ }^{3}$ Unidad de Cuidados Intensivos Neonatales; ${ }^{4}$ Psicología 2, Departamento de Seguimiento Pediátrico; ${ }^{5}$ Psicología 1, Departamento de Seguimiento Pediátrico; ${ }^{6}$ Comunicación Humana, Departamento de Seguimiento Pediátrico; ${ }^{7}$ Unidad tocoquirúrgica. Instituto Nacional de Perinatología. Ciudad de México, México

\section{Resumen}

Introducción: El gateo representa la primera forma de locomoción autónoma. Se han mencionado las implicaciones de la adquisición del gateo para lograr la marcha independiente y el control motor en el niño, pero son pocos los estudios relacionados con el gateo y sus efectos en el niño de alto riesgo biológico. Por eso se intentó conocer la relación entre la adquisición del gateo y la marcha independiente en una población de niños nacidos de alto riesgo en un programa de seguimiento pediátrico. Material y métodos: Estudio observacional, retrospectivo y descriptivo de una cohorte de niños de alto riesgo que acuden al seguimiento pediátrico, en el cual se revisó el periodo de adquisición del patrón de gateo y la marcha independiente. Resultados: Se integraron cuatro grupos: gateo normal, gateo limítrofe, gateo con retraso y gateo nulo. Se estudió a 558 lactantes; los grupos se integraron con gateo normal, 238 niños; gateo limítrofe, 96 lactantes; retraso en la adquisición del gateo, 207 niños; y gateo nulo, 17 niños. Por género, las niñas gatean mejor, con peso y edad gestacional mayores y predominio en los gateadores. La escala de Bayley señala mejores puntuaciones para los gateadores. En los niños con gateo normal, la marcha independiente se adquirió en el periodo normal a diferencia del grupo con retraso en el gateo en el cual la marcha independiente apareció con retraso. Conclusiones: En este estudio se identificó una relación entre la edad de inicio del gateo con la edad de adquisición de la marcha independiente, con mejor evolución en los niños gateadores.

Palabras clave: Gateo. Marcha. Alto riesgo. Bayley. Escala mental. Escala motora.

\section{Relationship between gait acquisition and independent gait in the high-risk child in follow-up}

\section{Abstract}

Background: Crawling represents the first form of autonomous human locomotion. Much has been said about the implications that an adequate acquisition of crawling has on development in order to achieve independent gait in the short term and the child's motor control in the long term. There are few studies related to crawling and its implications in children who were high biologic risk newborns. Therefore, we wanted to know the relation between crawling acquisition and independent gait in a

\section{Correspondencia:}

*María del P. Ibarra-Reyes

E-mail: pilaribarra_neurodesarrollo@ hotmail.com
Fecha de recepción: 10-06-2019

Fecha de aceptación: 16-08-2021 DOI: 10.24875/PER.19000048
Disponible en internet: 28-12-2021 Perinatol Reprod Hum. 2021;35(1):23-30

www.perinatologia.mx 0187-5337/@ 2021. Instituto Nacional de Perinatología Isidro Espinosa de los Reyes. Publicado por Permanyer. Este es un artículo open access bajo la licencia CC BY-NC-ND (http://creativecommons.org/licenses/by-nc-nd/4.0/). 
population of children who were high risk at birth in our Pediatric Follow up clinic. Material and Methods: An observational, retrospective, and analytical study of a cohort of children who were high risk at birth, and attended our pediatric follow-up clinic was done. The period between crawling acquisition and independent gait was reviewed. Results: 4 groups were integrated; normal crawling, borderline crawling, delayed crawling and null crawling. 558 infants were studied; the groups were integrated by: normal crawling 238 children; borderline crawling with 96 infants; delayed crawling with 207 children and null crawling with 17 children. By gender distribution, girls achieved better crawling. Weight and higher gestational age predominated in children with normal crawling. Crawlers had the best scores in the Bayley Scale. In children with normal crawling, independent gait was acquired within the normal period unlike the group with delay in crawling where independent gait was behind. Conclusions: In this study, we found a relation between the age of onset of crawling with the age of acquisition of the independent gait, with better skill in children who crawled.

Key words: Crawling. Gait. Risk higth. Bayley. Mental scale. Motor scale.

\section{Introducción}

El neurodesarrollo infantil es un proceso complejo y multivariado que depende de la maduración del sistema nervioso central y está determinado por modelos genéticos y la estimulación del medio; las adquisiciones conductuales se suceden en un orden establecido ${ }^{1}$. Cuando este se modifica por factores tan impactantes como la prematurez y el bajo peso, el niño puede sufrir un cambio radical en la adquisición y la expresión de las diversas habilidades esperadas para cada intervalo de edad.

El desarrollo psicomotor se refiere a una serie de sucesos cualitativos observados en los planos corporal, cognitivo y emocional, cuya adquisición ocurre de forma natural, secuencial y progresiva durante la infancia, por lo que adquirir una nueva habilidad requiere la consecución de habilidades previas. Aunque el desarrollo debe tener una secuencia ordenada y temporalidad similar en todos los niños, el logro de los diferentes hitos puede variar por múltiples factores biológicos y medioambientales ${ }^{2,3}$. Dentro del desarrollo normal aparecen hitos motores, es decir, el logro motor que se desarrolla de manera sistemática y que prepara al niño para emprender la siguiente habilidad ${ }^{4}$.

El desarrollo neuromotor es el proceso de evolución perceptivo motriz del lactante, a través de la estrecha relación entre el desarrollo neurológico y la adquisición de las habilidades motoras básicas, una capacidad motora en una secuencia de respuestas motoras precisas y una secuencia de aparición específica y ordenada. Las habilidades motoras básicas inician con el control cefálico y terminan con la marcha independiente, lo que da paso a capacidades complejas ${ }^{5}$.

Entre las habilidades motoras básicas, el gateo representa el primer patrón de locomoción autónoma que el niño adquiere, es el desplazamiento a través de una posición cuadrúpeda que representa la oportunidad de adquirir esquemas básicos de movimiento para la maduración motora; se inicia entre los seis y ocho meses y se extiende hasta los 11 meses $^{3-8}$.

El gateo incluye cambios en los aspectos perceptual, cognitivo y emocional. La mayoría inicia la movilidad mediante un desplazamiento hacia delante con arrastre sobre el estómago; esta acción lleva al cuerpo a lo largo de la superficie de apoyo mediante la actividad coordinada de brazos y piernas, y se desarrolla a partir de los giros, o la posibilidad de desplazar el cuerpo entre uno y otro decúbito 9 .

El gateo como primer movimiento armónico, sistemático y coordinado permite el establecimiento de conexiones entre los hemisferios cerebrales. Con el gateo, el niño desarrolla el control de tronco y pelvis para lograr la verticalización del cuerpo, al transitar por el patrón de hincado, semihincado y parado. Hace posible la disociación entre el movimiento de cintura escapular y cintura pélvica, además de la exploración del entorno, lo que fortalece el reconocimiento del espacio concreto, base del futuro manejo del espacio gráfico, y al mismo tiempo se refuerza el sistema de coordinación entre ojo-mano ${ }^{6,8-12}$.

Gesell1,3,9,13-20 y otros autores, aunque con discreta variación, determinan la edad de adquisición del gateo en el intervalo de siete a 10 meses; se avanza de modo inicial desde una posición estática en manos y rodillas hasta gatear con pericia y coordinación, tras dominar la transición a cuatro puntos de apoyo y regresar a la sedestación; a las 52 semanas se puede observar un gateo transitorio en manos y pies, una postura plantígrada que constituye la transición final hacia la postura erguida, de tal forma que a los 12 meses se inicie la marcha independiente. De igual modo, la marcha muestra un intervalo entre los 12 y 15 meses para la adquisición adecuada.

Diversos autores se han referido a la importancia de la adquisición del gateo y cómo la pobre tolerancia en 
el decúbito prono puede limitar su presencia o modificar su expresión ${ }^{21}$.

Además, su adquisición está influida por la decisión de no poner al niño boca abajo por el riesgo de muerte súbita ${ }^{22}$. Diversos factores biológicos, psicológicos y sociales pueden afectar el curso normal del desarrollo, por lo cual la vigilancia en el lactante con alto riesgo debe ser estrecha. Se define como niño de alto riesgo al lactante que, en virtud de sus antecedentes prenatales, perinatales o posnatales, tiene más probabilidades de sufrir, en los primeros años de vida, problemas del desarrollo, que pueden ser motores, cognitivos, sensoriales, lingüísticos o del comportamiento, y ser transitorios o permanentes ${ }^{23}$. La prematurez y el bajo peso determinan los factores de riesgo con mayores efectos en el desarrollo.

No se ha encontrado en las publicaciones médicas información específica de la adquisición del gateo y la marcha en la población de alto riesgo al nacimiento, con prematurez y bajo peso al nacimiento.

El Servicio de Estimulación Neuromotora del Departamento de Seguimiento Pediátrico del Instituto Nacional de Perinatología ha realizado por más de 30 años la vigilancia y la intervención temprana del neonato de alto riesgo biológico, es decir, niños nacidos antes de las 34 semanas de gestación, menores de 1,500 g, que desarrollan alguna afección neonatal: displasia broncopulmonar, hemorragia intraventricular, retraso en el crecimiento intrauterino, crisis convulsivas, neuroinfección, asfixia perinatal o ventilación mecánica $>72 \mathrm{~h}$.

A través de la evaluación periódica y con programas de intervención temprana se ha intentado promover un adecuado desarrollo neuromotor y psicomotor en el lactante y escolar que asisten al programa.

El objetivo del estudio es conocer la relación entre la adquisición del gateo con la marcha independiente en el niño de alto riesgo en seguimiento, así como la edad de adquisición promedio del gateo y la marcha en esta población, además de su relación con la escala del desarrollo de Bayley.

\section{Material y métodos}

Para esta investigación sobre la adquisición del gateo y su relación con la integración de la marcha independiente se condujo un estudio observacional, retrospectivo y descriptivo de una cohorte de niños de nacimiento de alto riesgo que acudieron al seguimiento pediátrico. Se revisaron los expedientes de 735 niños que se presentaron en el Servicio de Estimulación Neuromotora del Departamento de Seguimiento
Pediátrico, en el periodo de 2010 a 2015, para evaluar la edad de adquisición del gateo y la marcha; se utilizó la valoración neuroconductual del lactante ${ }^{24}$, un instrumento de tamiz, que tiene como objetivo la detección temprana de riesgos de retraso en el desarrollo y que se fundamenta en la observación del trabajo directo con el niño sobre los aspectos relevantes del desarrollo infantil. Se aplica en cortes en los meses 1, 4, 8, 12, 18 y 24 . Incluye 60 conductas, 10 reactivos por edad, y la elaboró Benavides en 1988. Este instrumento tiene amplia cobertura, califica si pasa o falla y arroja un resultado de normal, retrasado o dudoso. Solo se tomó el registro de la habilidad motora básica de gateo que se valora con el reactivo 5 del corte de los 12 meses y el reactivo 6 de la marcha del corte de los 18 meses. La valoración la aplicó siempre el mismo evaluador.

Se usó la escala del desarrollo infantil de Bayley $\|^{25}$, que valora el desarrollo funcional del niño y comprende dos subescalas: Índice de Desarrollo Mental (IDM) e Índice de Desarrollo Psicomotor (IDP). La escala la aplicaron dos psicólogas certificadas y el resultado se cataloga como:

- 115: desarrollo acelerado

- 84-114: desarrollo normal

- 78-83: ligero retraso en el desarrollo

- <69: desempeño significativamente retardado

Los criterios de inclusión fueron asistir al Servicio de Estimulación Neuromotora en el periodo comprendido entre enero de 2010 y diciembre de 2015, y contar con la evaluación de los 12 y 18 meses de la valoración neuroconductual.

Los criterios de exclusión fueron abandonar el programa antes de los 18 meses y no contar con algunas de las valoraciones a los 12 o 18 meses de edad. La muestra se dividió en cuatro grupos, tras considerar los intervalos de adquisición del gateo consignados en las publicaciones médicas y en los instrumentos de evaluación utilizados, en los que se espera la adquisición del gateo entre los 9 y 11 meses; el límite para la integración es a los 12 meses; y definitivamente el retraso en la capacidad después de los 13 meses de edad.

- Grupo 1, gateo normal: niños que gatearon antes de los 11 meses de edad cronológica.

- Grupo 2, gateo limítrofe: aquellos niños que lograron el patrón de gateo en el límite de los 12 meses de edad cronológica.

- Grupo 3, gateo con retraso: niños que gatearon después de los 13 meses de edad.

- Grupo 4, gateo nulo: niños que no adquirieron la capacidad de gatear. 
Para clasificar a los niños de acuerdo con el desarrollo de la marcha se definieron como sigue:

- Marcha normal, si esta se adquirió antes de los 17 meses de edad.

- Marcha en el límite de la integración normal, a los 18 meses.

- Marcha con retraso, después de los 19 meses de edad.

- Marcha nula o ausente.

Todos los niños del estudio están incluidos en un programa de intervención temprana del desarrollo, con evaluaciones trimestrales del desarrollo y actividades del neurodesarrollo en casa para la promoción de las habilidades motoras básicas.

Se analizó cada grupo en relación con la edad gestacional y el peso al nacimiento; la edad gestacional se clasificó como sigue:

- Prematuro extremo: <28 semanas.

- Muy prematuro: 28.1 a 32 semanas.

- Prematuro moderado o tardío: 32.1 a 37 semanas.

- Término: >37 semanas ${ }^{26}$.

El peso al nacimiento se clasificó en:

- Extremadamente bajo: peso <1,000 g.

- Peso muy bajo: 1,001 a 1,500 g.

- Peso bajo: 1,501 a 2,500 g ${ }^{27}$.

\section{Resultados}

Se estudió a 735 lactantes que asistieron al Servicio de Estimulación Neuromotora del Departamento de Seguimiento Pediátrico del INPer. Se excluyó a 177 por no satisfacer los criterios de inclusión. La muestra final fue de 558 lactantes, 290 (52\%) del sexo femenino y $268(48 \%)$ del masculino. La edad gestacional menor fue de 25 semanas y el límite mayor de 41 semanas, con una media de 30.5 semanas. El peso al nacimiento menor fue de $490 \mathrm{~g}$ y el mayor de $3,600 \mathrm{~g}$; la media fue de $1337.20 \mathrm{~g}$. El tipo de nacimiento fue de $520(93.2 \%)$ por vía abdominal y $38(6.8 \%)$ por vía vaginal. Al realizar la distribución de gateo por grupos se ubicó a 238 niños (42.7\%) en el grupo 1 de gateo normal; en el grupo 2 se incluyó a 96 niños (17.2\%) que adquirieron el gateo en el límite; en el grupo 3 de retraso en la adquisición del gateo a 207 niños (37.1\%) y en el grupo 4 a 17 lactantes (3.0\%) con gateo nulo. En la distribución por género, la mayor cantidad de niñas, el $47.2 \%$ se incluyó en el grupo 1 de gateo normal, a diferencia del $39.6 \%$ de niños que se alojaron en el grupo 3 de retraso en el gateo $\left(\chi^{2}\right.$ de Pearson, $p=0.132)$. En relación con la edad gestacional por grupos, se encontró una diferencia de dos semanas en relación con el grupo 1 de gateo normal con los otros grupos; asimismo, en la distribución por peso, la diferencia fue de 200 a casi $400 \mathrm{~g}$ del grupo normal para los grupos limítrofe, retraso y nulo (Tabla 1).

En cuanto a la edad gestacional se encontró que en el grupo 1 de gateo normal, el porcentaje fue mayor de prematuros moderados o tardíos. En el grupo 2 de gateo en el límite, el porcentaje mayor fue de niños muy prematuros (52.1\%). En el grupo 3 de retraso en la adquisición del gateo, el porcentaje mayor fue de productos muy prematuros; y en el grupo 4 de gateo nulo fue mayor la cantidad de prematuros moderados o tardíos ( $\chi^{2}$ de Pearson, $p=0.000$ ) (Tabla 2).

Cuando se observan los hallazgos en el peso al nacimiento, en todos los grupos el porcentaje mayor se registró en la categoría de 1,001 a $1,500 \mathrm{~g}$ ( $\chi^{2}$ de Pearson, $p=0.000$ ) (Tabla 3).

Respecto del gateo con la adquisición de la marcha independiente, se encontró lo siguiente: en el grupo 1 de gateo normal, $42.7 \%$ de los niños tuvo gateo normal, el $92.8 \%$ adquirió la marcha en el periodo normal, el $4.2 \%$ lo hizo en el periodo limítrofe y el $2.9 \%$ mostró retraso en la adquisición de la marcha. En el grupo 2 de gateo limítrofe de $17.2 \%$, el $73.9 \%$ adquirió la marcha adecuadamente, el $19.7 \%$ de niños lo hizo en el periodo límite y en el $6.2 \%$ la marcha se presentó con retraso. En el grupo 3 de gateo con retraso, del $37.1 \%$ de los niños con retraso en el gateo, el $21.2 \%$ de los niños adquirió la marcha en tiempo y forma adecuados y el $14.4 \%$ de los niños lo hizo en el periodo límite. El $63.7 \%$ de los niños tuvo retraso en la marcha y $0.48 \%$ no adquiró la marcha en el periodo del estudio. Del grupo 4 de gateo nulo, el $29.4 \%$ de los niños alcanzó la marcha en el periodo normal y el $15.8 \%$ en el periodo límite de adquisición. El 23.5\% alcanzó la marcha con retraso y el $41.1 \%$ no lo hizo ( $\chi^{2}$ de Pearson, $p=0.000$ ). A siete niños del grupo de gateo nulo después de los 12 meses se les diagnosticó parálisis cerebral infantil; tan solo uno de ellos logró la marcha independiente de tipo parético luego de los tres años de edad (Tabla 4).

En relación con los 17 niños del grupo 4 con gateo nulo se encontró que en ocho de ellos se había establecido la parálisis cerebral infantil de tipo cuadriparesia espástica; siete niños utilizaron andador (andadera comercial) por decisión de los padres y dos niños eran de madres solteras y cuidados por familiares, casos en los que la madre no supo si se estimulaba el gateo 0 usaban andador.

Cuando se analizaron los resultados obtenidos en la Escala de Desarrollo de Bayley a los 12 meses, en el 
Tabla 1. Variables demográficas

\begin{tabular}{|c|c|c|c|c|c|}
\hline Gateo & Normal & Límite & Retrasado & Nulo & Valor $p=$ \\
\hline $\mathrm{n}=558(100 \%)$ & $238(42.7 \%)$ & $96(17.2 \%)$ & $207(37.1 \%)$ & $17(3.0 \%)$ & \\
\hline Femenino, $290(52.0 \%)$ & $137(57.6 \%)$ & $45(46.9 \%)$ & $101(48.8 \%)$ & $7(41.2 \%)$ & 0.132 \\
\hline Masculino, $268(48.0 \%)$ & $101(42.4 \%)$ & $51(53.1 \%)$ & $106(51.2 \%)$ & $10(58.8 \%)$ & \\
\hline Edad gestacional, 31.18, Mn 25, Mx 41, DE 2.8 & $32.2 \mathrm{~s}$ & $30.7 \mathrm{~s}$ & $30.1 \mathrm{~s}$ & $30.4 \mathrm{~s}$ & 0.000 \\
\hline Peso, 1,344.9 g, Mn $490 \mathrm{~g}, \mathrm{Mx} 3,600 \mathrm{~g}$ & $1,507.2 \mathrm{~g}$ & $1,303.3 \mathrm{~g}$ & $1,190.1 \mathrm{~g}$ & $1,194.0 \mathrm{~g}$ & 0.000 \\
\hline Apgar 1 & 8 & 7 & 7 & 4 & 0.000 \\
\hline Apgar 5 & 9 & 9 & 9 & 8 & 0.000 \\
\hline Nac. vía abdominal & $223(93.7 \%)$ & $91(94.8 \%)$ & $192(92.8 \%)$ & $14(82.4 \%)$ & 0.297 \\
\hline Nac. vía vaginal & $15(6.3 \%)$ & $5(5.2 \% \%)$ & $15(7.2 \% \%)$ & $3(17.6 \%)$ & \\
\hline
\end{tabular}

Tabla 2. Gateo y edad gestacional

\begin{tabular}{|l|c|c|c|c|c|}
\hline Gateo & Normal & Límite & Retrasado & Nulo & Valor $\mathrm{p}=$ \\
\hline $\mathrm{n}=558(100 \%)$ & $238(100 \%)$ & $96(100 \%)$ & $207(100 \%)$ & $17(3.0 \%)$ \\
\hline$<28$ sem prematuro extremo, $110(19.7 \%)$ & $21(8.8 \%)$ & $21(21.9 \%)$ & $64(30.9 \%)$ & $4(23.5 \%)$ & 0.000 \\
\hline $28.1-32$ sem muy prematuro, $256(45.9 \%)$ & $101(42.4 \%)$ & $50(52.1 \%)$ & $98(47.3 \%)$ & $7(41.2 \%)$ \\
\hline $32.1-<37$ sem prematuro moderado 0 tardío, $177(31.7 \%)$ & $106(44.5 \%)$ & $24(25.0) \%$ & $41(19.8 \%)$ & $6(35.3 \%)$ \\
\hline$>37.1$ sem término, $15(2.7 \%)$ & $10(4.2 \%)$ & $1(1.0 \%)$ & $4(1.9 \%)$ & $0(0 \%)$
\end{tabular}

$\chi^{2}$ de Pearson, $p=0.000$.

Tabla 3. Gateo y peso al nacimiento

\begin{tabular}{|l|c|c|c|c|c|}
\hline Gateo & Normal & Límite & Retrasado & Nulo & Valor $\mathrm{p}=$ \\
\hline $\mathrm{n}=558(100 \%)$ & $238(42.7 \%)$ & $96(17.2 \%)$ & $207(37.1 \%)$ & $17(3.0 \%)$ & 0.000 \\
\hline$<1,000$ g extremadamente bajo & $27(11.3 \%)$ & $24(25.0 \%)$ & $80(38.6 \%)$ & $6(35.2 \%)$ & $8(47.0 \%)$ \\
\hline $1001-1,500 \mathrm{~g}$ peso muy bajo & $106(44.5 \%)$ & $46(47.9 \%)$ & $91(43.9 \%)$ & $8 \%$ \\
\hline $1501-2,500$ g peso bajo & $92(38.6 \%)$ & $23(23.9 \%)$ & $32(15.4 \%)$ & $3(17.6 \%)$ \\
\hline$>2,501$ g peso normal & $13(5.4 \%)$ & $3(3.1 \%)$ & $4(20.0 \%)$ & $0(0.0 \%)$ & \\
\hline
\end{tabular}

$\chi 2$ de Pearson, $\mathrm{p}=0.000$.

Índice de Desarrollo Mental los gateadores del grupo 1 alcanzaron las puntuaciones en el desarrollo acelerado y normal más altas de toda la población, aunque también en el desarrollo significativamente retardado la puntuación fue la mayor respecto de los otros grupos. En cuanto a los resultados obtenidos en el Índice de
Desarrollo Psicomotor, predominaron las puntuaciones altas en todas las clasificaciones del desarrollo en el grupo 1 de gateo normal, a excepción de la puntuación más alta de la clasificación del desarrollo significativamente retrasado que se ubicó en el grupo 3 de retraso en el gateo ( $\chi^{2}$ de Pearson, $p=0.022$ ) (Tabla 5). 
Tabla 4. Gateo y relación con la marcha

\begin{tabular}{|l|c|c|c|c|c|}
\hline & $\begin{array}{c}\text { Grup } 1 \\
\text { Gateo normal } 238\end{array}$ & $\begin{array}{c}\text { Grupo } 2 \\
\text { Gateo limítrofe } 96\end{array}$ & $\begin{array}{c}\text { Grupo } 3 \\
\text { Gateo retrasado 207 }\end{array}$ & $\begin{array}{c}\text { Grupo } 4 \\
\text { Gateo nulo } 17\end{array}$ & Valor $p=$ \\
\hline Marcha normal & $221(92.8 \%)$ & $71(73.9 \%)$ & $44(21.2 \%)$ & $5(29.5 \%)$ & 0.000 \\
\hline Marcha limítrofe & $10(4.2 \%)$ & $19(19.7 \%)$ & $30(14.4 \%)$ & $1(5.8 \%)$ \\
\hline Marcha retrasada & $7(2.9 \%)$ & $6(6.2 \%)$ & $132(63.7 \%)$ & $4(23.5 \%)$ \\
\hline Marcha nula & $0(0.0 \%)$ & $0(0.0 \%)$ & $1(0.48 \%)$ & $7(41.1 \%)$ \\
\hline
\end{tabular}

Tabla 5. Gateo y Escala de Bayley (12 meses)

\begin{tabular}{|c|c|c|c|c|c|}
\hline Gateo 12 m & $\begin{array}{l}\text { Normal } \\
238(100 \%)\end{array}$ & $\begin{array}{c}\text { Límite } \\
96(100 \%)\end{array}$ & $\begin{array}{l}\text { Retrasado } \\
207(100 \%)\end{array}$ & $\begin{array}{c}\text { Nulo } \\
17(100 \%)\end{array}$ & Valor $p=$ \\
\hline $\begin{array}{l}\text { IDM } \\
\text { No evaluados } \\
\text { Desarrollo acelerado } \\
\text { Desarrollo dentro de límites normales } \\
\text { Ligero retraso en el desarrollo } \\
\text { Desarrollo significativamente retrasado }\end{array}$ & $\begin{array}{c}59(24.8 \%) \\
12(5.0 \%) \\
105(44.1 \%) \\
37(15.5 \%) \\
25(10.5 \%)\end{array}$ & $\begin{array}{c}20(20.8 \%) \\
1(1.0 \%) \\
49(51.0 \%) \\
13(13.5 \%) \\
13(13.5 \%)\end{array}$ & $\begin{array}{c}56(27.1 \%) \\
4(1.9 \%) \\
93(44.9 \%) \\
31(15.0 \%) \\
23(11.1 \%)\end{array}$ & $\begin{array}{l}7(41.2 \%) \\
0(0.0 \%) \\
5(29.4 \%) \\
1(5.9 \%) \\
4(23.5 \%)\end{array}$ & 0.303 \\
\hline $\begin{array}{l}\text { IDP } \\
\text { Desarrollo acelerado } \\
\text { Desarrollo dentro de límites normales } \\
\text { Ligero retraso en el desarrollo }\end{array}$ & $\begin{array}{c}9(3.8 \%) \\
55(23.1 \%) \\
68(28.6 \%)\end{array}$ & $\begin{array}{c}2(2.1 \%) \\
22(22.9 \%) \\
22(22.9 \%)\end{array}$ & $\begin{array}{l}1(0.5 \%) \\
43(20.8 \%) \\
39(18.8 \%)\end{array}$ & $\begin{array}{c}0(0.0 \%) \\
1(5.9 \%) \\
5(29.4 \%)\end{array}$ & 0.022 \\
\hline $\begin{array}{l}\text { IDM } \\
\text { No evaluados }\end{array}$ & 47 (19.7\%) & $30(31.2 \%)$ & $68(32.9 \%)$ & $4(22.5 \%)$ & \\
\hline
\end{tabular}

\section{Discusión}

Existen muy pocos estudios sobre la adquisición del gateo y su relación con la marcha independiente en forma tan específica; algunos se refieren más a las cualidades del gateo ${ }^{25}$, la tolerancia del niño en la posición prona como promotora de control y la movilidad durante el primer año de vida ${ }^{28}$.

Ávila Aburdene ${ }^{29}$, en su estudio de los efectos de la utilización del andador, menciona que el gateo es un factor protector para desarrollar la marcha. Gunfrid ${ }^{30}$ señala que desplazarse en las manos y las rodillas se vincula con un inicio más temprano de caminar.

En su estudio, Karen ${ }^{31}$ afirma que la edad de inicio del gateo se relacionaba moderadamente con la edad de inicio del caminar; si el gateo comenzaba a edades tempranas, también lo hacía la caminata a edad menor. La experiencia de gatear prepara a los lactantes para percibir las posibilidades cuando comienzan a caminar.

Farber ${ }^{32}$ concede importancia a la experiencia del gateo temprano en el desarrollo de los sistemas sensoriales y motores en el niño. McEwan ${ }^{33}$ encontró también la importancia del gateo en el desarrollo temprano y ya desde Piaget se consideraba que la locomoción autónoma era fundamental para los cambios posturales de la primera infancia, que el gateo desencadenaba en particular la base para la percepción visual espacial y que dicha experiencia contribuía a la adecuada integración sensorial del niño. El proceso de gateo proporciona coordinación ojo-mano, procesamiento vestibular, el equilibrio y la conciencia espacial, la aferencia táctil y la conciencia cinestésica. También encontró que la ausencia de gateo se relacionaba con puntuaciones más bajas en pruebas de desarrollo; cabe resaltar que en su estudio se integró a niños sin antecedentes de riesgo.

En relación con este estudio, los hallazgos fueron relevantes: las niñas mostraron un desarrollo psicomotor más adecuado respecto de los niños; el $47.2 \%$ de las niñas alcanzó el gateo en tiempo y forma en comparación con el $37.7 \%$ de los niños.

Fue significativo que la edad de gestación y el peso mayor influyen para un mejor desarrollo; en la 
distribución por grupos y en el de gateo normal, la diferencia fue de dos semanas mayor en relación con los otros grupos de análisis. Fue significativo que el niño prematuro extremo desarrolló retraso en el gateo a diferencia de los prematuros moderados y de los tardíos. En los niños nacidos a término también fue mayor el porcentaje con gateo normal.

El peso también es un factor determinante para el desarrollo; en el grupo 1 de gateo normal, la diferencia fue de $200 \mathrm{~g}$ con el grupo de gateo en el límite, pero de $300 \mathrm{~g}$ con los grupos de retraso y gateo nulo. Con peso mayor de 1,001 g es más fácil alcanzar un patrón de gateo normal y con peso extremadamente bajo los resultados arrojaron mayor porcentaje de retraso en la adquisición del gateo.

Para la adquisición de la marcha independiente, los niños gateadores del grupo 1 alcanzaron la habilidad sin problema y, en el periodo de tiempo esperado, el $92.8 \%$ del grupo. En el grupo limítrofe, la marcha en el $73.9 \%$ también se adquirió dentro del intervalo normal; a diferencia de los niños que presentaron retraso, este persistió en la marcha en un $63.7 \%$.

En el $57.5 \%$ de la muestra, los niños con gateo normal y en el límite adquirieron la marcha independiente sin problema; solo el $8.07 \%$ de estos grupos presentó retraso. Esto contrasta con el $14.3 \%$ de los grupos de retraso y gateo nulo que lograron la marcha independiente, con el $25.8 \%$ en los que persistió el retraso de la marcha. Asimismo, se observaron bajas puntuaciones en los niños no gateadores en la Escala de Desarrollo de Bayley, de manera específica en el Índice de Desarrollo Psicomotor.

\section{Conclusiones}

En el Servicio de Estimulación Neuromotora de Seguimiento Pediátrico del Instituto Nacional de Perinatología se identificó en este estudio que si el patrón de gateo se adquiere en tiempo y forma adecuados, la marcha independiente se presenta en el momento esperado y con calidad adecuada, a diferencia de los niños que no logran el gateo en un periodo esperado, en quienes la marcha independiente también se consiguió en forma tardía. En este estudio se observó que las niñas tienen asimismo una mejor adquisición del gateo y la marcha en relación con los niños, del mismo modo que el peso y la edad gestacional al nacimiento contribuyen a la adquisición en tiempo adecuado del gateo. Esto no fue significativo estadísticamente en el Índice de Desarrollo Mental de la Escala de Bayley, pero sí en los resultados del Índice de Desarrollo Psicomotor.

El gateo es una capacidad motora determinante para alcanzar la autonomía motora, en la que los profesionales de la salud y la educación al cuidado del niño no deben escatimar esfuerzos. Los niños de alto riesgo deben conseguir de forma adecuada el patrón porque a largo plazo será la base para la apropiada consecución de la marcha y de muchas otras funciones indispensables en el neurodesarrollo infantil. Será interesante en el futuro replicar este estudio con una población más grande y que no haya recibido vigilancia en un seguimiento pediátrico para determinar si la intervención temprana es un elemento que facilita la crianza y el desarrollo.

\section{Financiamiento}

La presente investigación no ha recibido ninguna beca específica de agencias de los sectores públicos, comercial, o con ánimo de lucro.

\section{Conflicto de intereses}

Los autores declaran no tener conflicto de intereses.

\section{Responsabilidades éticas}

Protección de personas y animales. Los autores declaran que para esta investigación no se han realizado experimentos en seres humanos ni en animales.

Confidencialidad de datos. Los autores declaran que han seguido los protocolos de su centro de trabajo sobre la publicación de datos de pacientes.

Derecho a la privacidad y consentimiento informado. Los autores han obtenido el consentimiento informado de los pacientes y/o sujetos referidos en el artículo. Este documento obra en poder del autor de correspondencia.

\section{Bibliografía}

1. Flehmig I. Desarrollo normal del lactante y sus desviaciones. Diagnóstico y tratamiento tempranos. Argentina: Médica Panamericana. Argentina, 1988.

2. Martínez C. Evaluación del desarrollo psicomotor de niños institucionalizados menores de 1 año mediante tres herramientas distintas de evaluación. [Tesis de grado] Santiago, Universidad de Chile; 2005 [cited 2012 Sep 24]; Available from: https://goo.gl/kr8Nzx.)

3. Sánchez PC, Rivera GR, Correa RA, Figueroa M, Sierra CA, Izazola SC. El desarrollo del niño hasta los 12 meses. Orientaciones al pediatra para su vigilancia con la familia. Acta Pediatr Mex 2015;36:480-496.

4. Morales SA, Rincón LCF. Relación entre madurez neuropsicológica y presencia-ausencia de conducta de gateo. Acta de Investigación Psicológica 2016;6:2450-2458 
5. Ibarra RMP. Estimulación neuromotora del niño con daño neurológico. En: Poblano A. Detección y estimulación tempranas del niño con daño neurológico. México. Editores de Textos Mexicanos, 2003.

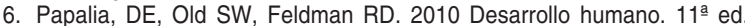
México: McGraw-Hill, 2010.

7. Vojta V, Scheweizer E. El descubrimiento de la motricidad ideal. España Ed. Morata, 2011.

8. Lipkin P. Motor development and disfunction. In: Developmental-Behavioral Pediatric. 4a 2009.

9. Gesell A, Amatruda C. Diagnóstico del desarrollo normal y anormal del niño. Evaluación y manejo del desarrollo neuropsicológico normal y anormal del niño pequeño y el preescolar. México. Ed. Paidós, 1985.

10. Hervas $\mathrm{AH}$. El desarrollo motor del niño desde su nacimiento hasta los 2 años. Revista Digital Investigación y Educación. 2006;25.

11. Villegas de Martínez M, Calarco B. Bebés prematuros y recién nacidos de término de riesgo. Su evaluación, seguimiento y propuestas de servicio. Rev Iberoam Fisioter Kinesiol. 2000;3(2):2-7.

12. Collado VS. Desarrollo de la marcha. Disponible en: http://www.aux.es/ publicaciones/archivos/CCSREV05_002.pdf CIENSALUD 2005

13. Hernández GM. Modelo dinámico para valoración del gateo. Rev Mex Med Fis Rehab. 2016;28(1-2):28-32.

14. Coutiño LB. Desarrollo psicomotor. Rev Mex Med Fis Reh. 2002;14:58-60

15. Roche HMC. Desarrollo psicomotor del niño de menor edad: una aproximación práctica. An Pediatr Contin. 2007;5(4):19-204.

16. Chico MIJ, Narváez PSK, Umaquinga CAC, Rosero MPD. Sistema embebido orientado a bebés en etapa de gateo para prevención de accidentes aplicado en un textil inteligente. Enfoque UTE, V9-N.1. 2018 217-225.

17. Medina AMP, Caro KI, Muñoz HP, Leyva SJ, Moreno CJ, Vega SSM Neurodesarrollo infantil: características normales y signos de alarma en el niño menor de cinco años. Rev Peru Med Exp Salud Pública. 2015;32(3):565-73.

18. Estimulación temprana. Lineamientos técnicos. México: Secretaría de Salud, 2002.
19. García PMA, Martínez GMA. Desarrollo psicomotor y signos de alarma. En: AEPap (ed.) Curso de actualización en pediatría 2016. Madrid: Lúa Ediciones 2016;30:81-93.

20. Gómez $\mathrm{AD}$, Pulido $\mathrm{VI}$, Fiz PL. desarrollo neurológico normal del niño. Pediatr Integral. 2015;XIX (9):640.e1-640.e7.

21. Kuo YL, Liao HF, Chen PC, Hsieh WS, Hwang AW. Influence of wakeful prone positioning on motor development during the early life. J Dev Behav Pediatr. 2008;29:367-376.

22. Majnemer A, Barr R. Association between sleep position and early motor development. J Pediatr. 2006;149:623-9.

23. Ramos SI, Márquez LA. Recién nacido de riesgo neurológico. Vox Paediatrica. 2000;8(5):5-10.

24. Benavides GHM, Fernández CLA, Venta SJA, Bravo CZ, Ibarra RMP, Barroso AJ. Utilidad de una valoración neuroconductual de tamiz en el primer año de vida. Perinatol Reprod Hum. 1989;3(4):193-8.

25. Bayley N. Bayley Scale of Infant Development. $2^{\text {nd }}$ ed. (BSID-II). San Antonio: Hartcourt Brace \& Company, 1993

26. Quinn JA. Preterm birth: case definition \& guidelines for data collection, analysis, and presentation of immunisation safety data. Vaccine. 2016. http: dx.doi.org/10.1016/j.vavvine.2016.03.045.

27. Mandy GT. Short-term complications of the preterm infant. In: Weisman LE, Kim MS, editors. UpToDate Waltham, M. A. 2016.

28. Guidetti J, Wells J, Worsdall A, Metz A. The effect of positional support on tolerancia of wakeful prone in infants. Physical \& Occupational Therapy in Pediatrics. 2016;1:1-14.

29. Ávila AR, Castro KM. Relaciones con el inicio de la marcha, gateo, uso de andaderas y accidentes. Rev Soc Bol Ped. 2005;44(1):11-14.

30. Gunfrid VS, Aarethun K, Bratberg GH. Age for onset of walking and prewalking strategies. Early Human Development. 2013;89:655-659.

31. Karen EA, Berger S, Leo AJ. Developmental continuity? Crawling, cruising, and walking. Developmental Science 2011;14(2):306-318.

32. Farber S. Neurohabilitation. Philadelphia: Saunders, 1982.

33. McEwan M, Dihoff R, Brosivc G. Early infant crawling experience in reflected in later motor skill development. Perceptual and Motor Skills. 1991;72:75-79. 